\title{
Constraining the population of cosmic ray protons in cooling flow clusters with $\gamma$-ray and radio observations: Are radio mini-halos of hadronic origin?
}

\author{
C. Pfrommer and T. A. Enßlin
}

\begin{abstract}
Max-Planck-Institut für Astrophysik, Karl-Schwarzschild-Str.1, Postfach 1317, 85741 Garching, Germany e-mail: pfrommer@mpa-garching.mpg.de
\end{abstract}

A\&A 413, 17-36 (2004), DOI: 10.1051/0004-6361:20031464

Key words. errata, addenda - galaxies: cooling flows - galaxies: clusters: general galaxies: clusters: individual: Perseus (A426) - radiation mechanisms: non-thermal

The normalization of the inverse Compton (IC) flux induced by secondary cosmic ray electrons (CRe) in Fig. 3 has been incorrectly computed. The correct ratio of $\gamma$-ray flux resulting from decaying pions relative to IC flux from secondary CRe is $1.5 \times 10^{-1}\left(\alpha_{\mathrm{p}}=2.1\right)$ and $1.7 \times 10^{-3}\left(\alpha_{\mathrm{p}}=2.7\right)$ at $E_{\gamma}=1 \mathrm{GeV}$. This is illustrated in Fig. 1, which replaces Fig. 3 of our paper.

This induces a minor change in the expected IC emission (cf. Sect. 4.1.) of hadronically originating CRe in the Perseus cluster. Assuming a cosmic ray proton (CRp) spectral index of $\alpha_{\mathrm{p}}=2.3$ and taking the CRp normalization obtained by comparing the hadronically induced synchrotron emission to the observed radio mini-halo of Perseus, the secondary IC emission ought to have read as

$\frac{\mathrm{d} \mathcal{F}}{\mathrm{d} E}(20 \mathrm{keV})=\mathcal{F}_{\mathrm{IC}} 10^{-7} \gamma \mathrm{cm}^{-2} \mathrm{~s}^{-1} \mathrm{keV}^{-1}$,

with $\mathcal{F}_{\mathrm{IC}}=8.4,4.2$, and 2.3 for $B_{0}=5 \mu \mathrm{G}, 10 \mu \mathrm{G}$, and $20 \mu \mathrm{G}$. Comparing these results to the post-launch spectral sensitivity of $4 \times 10^{-6} \gamma \mathrm{s}^{-1} \mathrm{~cm}^{-2} \mathrm{keV}^{-1}$ to the continuum at $20 \mathrm{keV}$ for an observation time of $10^{6} \mathrm{~s}(3 \sigma$ detection), there is only a minor chance to detect IC emission of CRe as previously concluded. All other figures, formulae, and conclusions remain unchanged.

\section{References}

Reimer, O., Pohl, M., Sreekumar, P., \& Mattox, J. R. 2003, ApJ, 588, 155

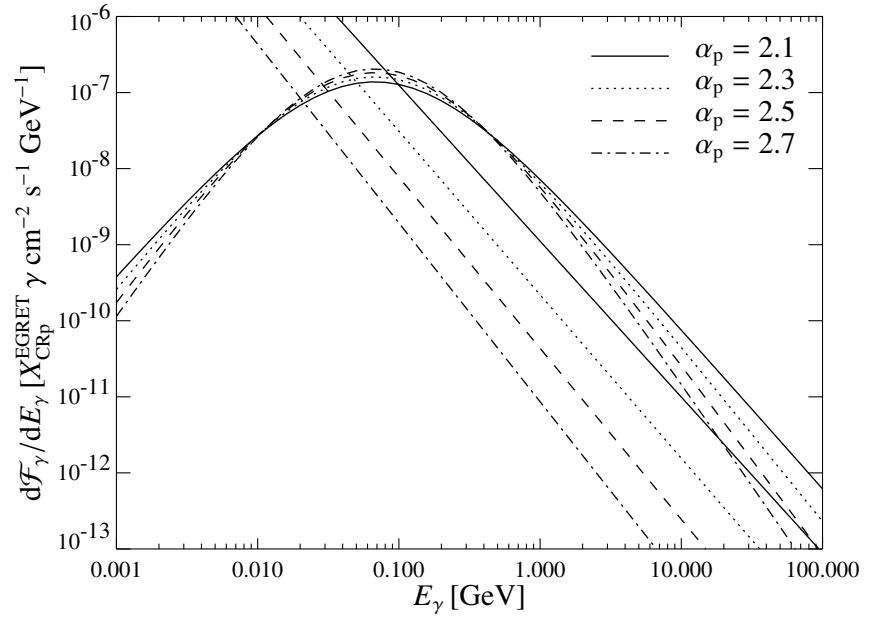

Fig. 1. The simulated differential flux of $\gamma$-rays from Perseus reaching the Earth. Shown are upper limits of the IC emission of secondary CRe (power-laws, assuming zero magnetic field) as well as pion decay induced $\gamma$-ray emission (represented by broad distribution centered on $E_{\text {peak }} \simeq 67.5 \mathrm{MeV}$ ). The normalization of the spectra differing in their values of the CRp spectral index $\alpha_{\gamma}=\alpha_{\mathrm{p}}$ (Dermer's model) depends on the assumed scaling between CRp and thermal energy density. We fix this scaling parameter $X_{\mathrm{CRp}}$ assuming the isobaric model by comparing the integrated flux above $100 \mathrm{MeV}$ to EGRET upper limits (see Reimer et al. 2003). 